\title{
Comunicación
}

\section{Frecuencia de Neoplasias en Caninos de 0 a 5 Años Diagnosticadas en el Laboratorio de Histopatología Veterinaria de la Universidad Nacional Mayor de San Marcos (2003-2014)}

\author{
Frequency of Neoplasms in Canines 0 to 5 Years of Age Diagnosed in the \\ Laboratory of Veterinary Histopathology of the National UniVERSity \\ of SAN Marcos (2003-2014)
}

\author{
Ghiis Chang H. ${ }^{1}$, Rosa Perales C., ${ }^{1,2}$ Luis Tabacchi N. ${ }^{1}$
}

\section{Resumen}

Se evaluaron un total de 4701 protocolos de caninos, procesados en el Laboratorio de Histopatología de la Facultad de Medicina Veterinaria de la Universidad Nacional Mayor de San Marcos (Lima) durante el periodo 2003-2014 para determinar la frecuencia de los tipos de neoplasias diagnosticadas mediante histopatología y en el caso del tumor venéreo transmisible (TVT) por citología. Se hallaron 3480 casos de neoplasias, de las cuales $554(11.8 \%)$ pertenecían a caninos de 0 a 5 años de edad. Los canes de 4 a 5 años (261 casos; 47.1\%) y los de sexo masculino (333 casos; 60.1\%) presentaron la mayor cantidad de neoplasias. Las neoplasias benignas predominaron con 279 casos $(50.4 \%)$, siendo el TVT la neoplasia más frecuente de este grupo y del conteo general con 94 casos (33.7\%). La neoplasia maligna más frecuente fue el rabdomiosarcoma, con 47 casos $(17.1 \%)$.

Palabras clave: neoplasia; frecuencia; caninos; edad

\section{Abstract}

A total of 4701 canine protocols, processed at the Laboratory of Histopathology of the Faculty of Veterinary Medicine of the National University of San Marcos (Lima) during the period 2003-2014 were evaluated to determine the frequency of types of cancer diagnosed by histopathology and in the case of the transmissible venereal tumor (TVT)

1 Laboratorio de Histología, Embriología y Patología Veterinaria, Facultad de Medicina Veterinaria, Universidad Nacional Mayor de San Marcos, Lima, Perú

${ }^{2}$ E-mail: rperalesc@unmsm.edu.pe

Recibido: 14 de noviembre de 2016

Aceptado para publicación: 30 de marzo de 2017 
by cytology. There were 3480 cases of neoplasms, of which $554(11.8 \%)$ belonged to canines from 0 to 5 years of age. The $4-5$ years old dogs (261 cases, $47.1 \%$ ) and the male dogs $(333$ cases, $60.1 \%)$ had the highest number of neoplasms. The benign neoplasms predominated with 279 cases $(50.4 \%$ ), with TVT being the most frequent neoplasia of this group and of the general count with 94 cases $(33.7 \%)$. The most frequent malignant neoplasm was rhabdomyosarcoma, with 47 cases (17.1\%).

Key words: neoplasm; frequency; canine; age

\section{INTRODUCCIÓN}

Las neoplasias caninas tienen gran importancia en medicina veterinaria por ser una patología frecuente y una de las principales causas de muerte en animales domésticos (Cartagena et al., 2011). El estudio de De Vivero et al. (2013) indica que su incidencia en el medio local se ha incrementado con el transcurrir de los años.

Dentro de los datos de la casuística veterinaria y la literatura, existen reportes que indican que algunas neoplasias, como el tumor venéreo transmisible, papiloma, mastocitoma y linfoma, tienen mayor incidencia en los caninos más jóvenes (Meuten, 2002; Morris, 2002). En estudios similares realizados en el Laboratorio de Histología, Embriología y Patología Veterinaria de la Facultad de Medicina Veterinaria de la Universidad Nacional Mayor de San Marcos (LHEPV-FMV-UNMSM), se ha evidenciado la presencia de neoplasias en las etapas más tempranas; no obstante, no existe información epidemiológica disponible que detalle los tipos de neoplasias que pueden aparecer durante los primeros años de vida en el país, debido a que dichos estudios no contemplaron animales de todos los grupos etarios (Schmidt et al., 2010; Chau et al., 2013). Este faltante amerita realizar estudios que relacionen el comportamiento biológico neoplásico a edades tempranas con variables de interés como grupo etario, raza, sexo y origen celular comprometido.

El presente tiene como objetivo determinar la frecuencia de neoplasias que afec$\tan$ a caninos de 0 a 5 años e identificar los tipos de neoplasias con mayor incidencia, según edad, sexo y raza, mediante la revisión de protocolos histopatológicos diagnosticados en el LHEPV-FMV-UNMSM.

\section{Materiales y Métodos}

En el estudio retrospectivo se utilizó como fuente de datos los informes obtenidos de protocolos de necropsia y exámenes independientes solicitando histopatologías y citologías (casos propios y provenientes de diversas clínicas y centros veterinarios de Lima), que fueron realizadas en el LHEPVFMV-UNMSM durante el periodo 20032014. Solo en el caso del tumor venéreo transmisible (TVT) se consideraron los informes citológicos, dado que es la técnica que mayormente se usa para los estudios referentes a esta neoplasia.

Se consideraron los datos de los individuos de 0 a 5 años de edad con neoplasias. Se tomaron en cuenta las siguientes variables: raza (pura o mestiza), edad (0-12, 1324, 25-36, 37-48, 49-60 meses), sexo (macho, hembra) y origen celular (epitelial, mixto, conectivo, hematopoyético, endotelial, muscular y reproductivo). 


\section{Resultados}

El LHEPV registró 4701 informes histopatológicos y citológicos correspondientes a caninos en el periodo del estudio, de los cuales $3480(74.0 \%)$ resultaron ser neoplasias y $554(11.8 \%)$ correspondieron a canes entre 0 a 5 años. De estas últimas, 473 (85.4\%) fueron halladas mediante histopatología y las restantes $81(14.6 \%)$ mediante citologías compatibles con TVT.

La mayor frecuencia de neoplasias se presentó en el grupo etario de 49-60 meses (261 casos; 47.1\%), seguido de los grupos de 25-36 meses (98 casos; 17.7\%), 37-48 meses (79 casos; $14.3 \%), 13-24$ meses (60 casos; $10.8 \%$ ) y de $0-12$ meses (56 casos; $10.2 \%$ ). Asimismo, los machos fueron más afectados (333 casos; 60.1\%) que las hembras (221 casos; 39.9\%).

Se diagnosticaron neoplasias en canes de 48 razas. La mayor frecuencia se presentó en los mestizos (142 casos; 25.6\%), seguidos de la raza Bóxer (66 casos; $11.9 \%$ ), Labrador (44 casos; 7.9\%), Golden Retriever (26 casos; 4.7\%), Cocker Spaniel y Schnauzer (24 casos cada uno, $4.3 \%$ ) y Rottweiler (23 casos; 4.2\%).

Las neoplasias más frecuentes fueron las epiteliales con 194 casos (35.0\%), seguidos de neoplasias de origen reproductivo (110 casos; 19.9\%), hematopoyéticas (71 casos; $12.8 \%$ ), conectivas ( 65 casos; $11.7 \%$ ), musculares (54 casos; 9.7\%), endoteliales (44 casos; $7.9 \%$ ) y mixtas (16 casos; $2.9 \%$ ).

De los 554 casos diagnosticados, 279 fueron neoplasias benignas (50.4\%) y 275 fueron malignas $(49.64 \%)$. Dentro de las neoplasias benignas, los tipos más frecuentes fueron de tipo reproductivo con $100 \mathrm{ca}-$ sos (35.8\%), epitelial con 82 casos (29.4\%), hematopoyético con 41 casos $(14.7 \%)$ y conectivo con 39 casos (14.0\%). La neoplasia benigna más frecuente fue el TVT con 94 casos (33.7\%), así como la neoplasia más frecuente de todas (benignas y malignas) (Cuadro 1).

En el caso de las neoplasias malignas (Cuadro 2), los tipos más frecuentes fueron epitelial con 104 casos (37.8\%), muscular con 54 casos (19.6\%), conectivo con 46 casos (16.7\%) y hematopoyético con 36 casos (13.1\%). Según el diagnóstico específico, el más frecuente fue el rabdomiosarcoma (tipo muscular) con $17.1 \%$ (47 casos); asimismo, se ubica como la segunda neoplasia más frecuente de todas (8.5\% del total).

\section{Discusión}

El 11.8\% de neoplasias en caninos de 0-5 años implica un aumento importante de neoplasias para este grupo etario (Díaz, 1990), solo comparable al estudio de Castro (1985) quién detectó $10.3 \%$ de este tipo de casos en caninos menores de 5 años de edad. Este incremento puede estar relacionado a una mayor disposición de los dueños para realizar exámenes médicos que permite una detección precoz de las neoplasias (Cartagena et al., 2011), así como al aumento de radiación UV y la contaminación ambiental en las ciudades (van den Hoven, 2011; Fajardo et al., 2013).

La gran cantidad de caninos afectados entre los 4 a 5 años confirma que a mayor edad hay también mayor incidencia de neoplasias (Morris, 2002). Asimismo, el estrato etario de 2 a 3 años coincide con la madurez sexual, lo cual predispone al contagio del TVT $(22.5 \%$ de neoplasias en este periodo).

La mayor cantidad de neoplasias en los machos difiere de los resultados de De Vivero et al. (2013) y San Martín (2005). Esta diferencia podría deberse a la mayor actividad sexual que presentan los machos y a la menor predisposición de las hembras jóvenes a desarrollar tumores mamarios (Chau et al, 2013). Por otro lado, la mayor frecuen- 
Cuadro 1. Número (n) y frecuencia (\%) de neoplasias benignas en caninos de 0 a 5 años, según su origen celular, diagnosticadas en el Laboratorio de Histopatología de la Facultad de Medicina Veterinaria, Universidad Nacional Mayor de San Marcos, Lima, durante el periodo 2003-2014

\begin{tabular}{|c|c|c|c|}
\hline \multirow{2}{*}{ Tipo } & \multirow{2}{*}{$\begin{array}{c}\text { Casos } \\
\text { (n) }{ }^{1}\end{array}$} & \multicolumn{2}{|c|}{ Frecuencia } \\
\hline & & $\% \%^{2}$ & $\%$ Total $^{3}$ \\
\hline Epitelial & 82 & 29.4 & 14.8 \\
\hline Adenoma & 30 & 10.8 & 5.4 \\
\hline Adenoma de glándula perianal & 6 & 2.2 & 1.1 \\
\hline Adenoma de glándula mamaria & 6 & 2.2 & 1.5 \\
\hline Adenoma de glándulas sebáceas & 5 & 1.8 & 0.9 \\
\hline Adenomas (otros) & 13 & 4.7 & 2.4 \\
\hline Epuli & 7 & 2.5 & 1.3 \\
\hline Papiloma & 31 & 11.1 & 5.6 \\
\hline Tricoepitelioma & 14 & 5.0 & 2.5 \\
\hline Mixto & 5 & 1.8 & 0.9 \\
\hline TMMB & 5 & 1.8 & 0.9 \\
\hline Conectivo & 39 & 14.0 & 7.0 \\
\hline Fibroma & 3 & 11 & 0.5 \\
\hline Mixoma & 0 & - & - \\
\hline Lipoma & 8 & 2.9 & 1.4 \\
\hline Mastocitoma benigno & 19 & 6.8 & 3.4 \\
\hline Histocitoma & 9 & 3.3 & 1.6 \\
\hline Hematopoyético & 41 & 14.7 & 7.4 \\
\hline Plasmocitoma cutáneo & 41 & 14.7 & 7.4 \\
\hline Muscular & 1 & 0.4 & 0.2 \\
\hline Leiomioma & 1 & 0.4 & 0.2 \\
\hline Endotelial & 11 & 3.9 & 2.0 \\
\hline Hemangioma & 9 & 3.2 & 1.6 \\
\hline Hemangiopericitoma & 1 & 0.4 & 0.2 \\
\hline Linfangioma & 1 & 0.4 & 0.2 \\
\hline Reproductivo & 100 & 35.8 & 18.1 \\
\hline Seminoma & 3 & 1.1 & 0.5 \\
\hline Tumor de células de Sertoli & 2 & 0.7 & 0.4 \\
\hline Tumor de células de Leydig & 1 & 0.4 & 0.2 \\
\hline Tumor venéreo transmisible (TVT) & 94 & 33.7 & 17.0 \\
\hline Total & 279 & 100 & 50.4 \\
\hline
\end{tabular}

${ }^{1}$ Número de casos diagnosticados

${ }^{2}$ Frecuencia expresada en porcentaje respecto al total de casos benignos

${ }^{3}$ Frecuencia expresada en porcentaje respecto al total de casos de neoplasia (benignos + malignos) 
Cuadro 2. Número (n) y frecuencia (\%) de neoplasias malignas en caninos de 0 a 5 años, según su origen celular, diagnosticadas en el Laboratorio de Histopatología de la Facultad de Medicina Veterinaria, Universidad Nacional Mayor de San Marcos, Lima, durante el periodo 2003-2014

\begin{tabular}{|c|c|c|c|}
\hline \multirow{2}{*}{ Tipo } & \multirow{2}{*}{$\begin{array}{c}\text { Casos } \\
\text { (n) }{ }^{1}\end{array}$} & \multicolumn{2}{|c|}{ Frecuencia } \\
\hline & & $\%{ }^{2}$ & $\%$ Total $^{3}$ \\
\hline Epitelial & 104 & 37.8 & 18.8 \\
\hline Carcinoma & 64 & 23.3 & 11.6 \\
\hline Carcinoma de células basales & 8 & 2.9 & 1.4 \\
\hline Carcinoma epidermoide (CCE) & 40 & 14.6 & 7.2 \\
\hline Carcinoma (otros) & 16 & 5.8 & 2.9 \\
\hline Adenocarcinoma & 40 & 14.6 & 7.2 \\
\hline Adenocarcinoma mamario & 11 & 4.0 & 2.0 \\
\hline Adenocarcinoma de gl. perianales & 0 & - & - \\
\hline Adenocarcinoma (otros) & 29 & 10.6 & 5.2 \\
\hline Mixto & 2 & 0.7 & 0.4 \\
\hline TMMM & 2 & 0.7 & 0.4 \\
\hline Conectivo & 46 & 16.7 & 8.3 \\
\hline Fibrosarcoma & 11 & 4.0 & 2.0 \\
\hline Mixosarcoma & 1 & 0.4 & 0.2 \\
\hline Condrosarcoma & 2 & 0.7 & 0.4 \\
\hline Osteosarcoma & 3 & 1.1 & 0.5 \\
\hline Liposarcoma & 5 & 1.8 & 0.9 \\
\hline Mastocitoma maligno & 9 & 3.3 & 1.6 \\
\hline Melanoma & 15 & 5.5 & 2.7 \\
\hline Hematopoyético & 36 & 13.1 & 6.5 \\
\hline Linfoma & 20 & 7.3 & 3.6 \\
\hline Plasmocitoma maligno & 14 & 5.1 & 2.5 \\
\hline Timoma & 2 & 0,7 & 0.4 \\
\hline Muscular & 54 & 19.6 & 9.8 \\
\hline Leiomiosarcoma & 7 & 2.6 & 1.3 \\
\hline Rabdomiosarcoma & 47 & 17.1 & 8.5 \\
\hline Endotelial & 33 & 12.0 & 6.0 \\
\hline Hemangiosarcoma & 32 & 11.6 & 5.8 \\
\hline Linfangiosarcoma & 1 & 0.4 & 0.2 \\
\hline Total & 275 & 100.0 & 49.6 \\
\hline
\end{tabular}


cia en canes mestizos sobre las razas puras probablemente se deba a la gran población de canes mestizos que hay en el medio.

La predominancia de neoplasias epiteliales concuerda con Díaz (1990) y Castro (1985) y la tendencia de las neoplasias reproductivas coincide con Takano (1964), Rodríguez (1978) y Castro (1985).

La mayor, pero ligera proporción de neoplasias benignas presenta un resultado inverso a otros estudios recientes hechos por este laboratorio, posiblemente debido a una mayor detección precoz de estas neoplasias. Además, en los primeros 5 años de edad se tiene una menor exposición a factores externos que pueden desencadenar la aparición o evolución de un cáncer maligno (Morris, 2002).

La alta frecuencia del TVT coincide con la teoría que relaciona la ocurrencia de este tumor con el auge de la actividad sexual (Takano, 1964; Rodriguez, 1978; Castro, 1985; Díaz, 1990; De Vivero et al., 2013). Por otro lado, la presencia del rabdomiosarcoma como la neoplasia maligna más frecuente discrepa con los estudios de San Martín (2005) y Díaz (1990), quienes colocan al carcinoma mamario como la neoplasia maligna más frecuente. Esta diferencia podría deberse a la epidemiología del adenocarcinoma mamario, que suele aparecer en hembras mayores de 6 años (Chau et al., 2013).

\section{Literatura Citada}

1. Cartagena J, Chacón F, Domingo V, Hervás J, Moya S. 2011. Oncología veterinaria. Zaragoza, España: Servet. 194 p.

2. Castro J. 1985. Frecuencia en la presentación de neoplasias en caninos periodo 197-1983. Tesis de Médico Veterinario. Lima: Univ Nacional Mayor de San Marcos. 46 p.
3. Chau G, Chavera A, Perales $R$, Gavidia C. 2013. Frecuencia de neoplasias en glándula mamaria de caninos: estudio retrospectivo en el periodo 1994-2006 en la ciudad de Lima, Perú. Rev Inv Vet Perú 24: 72-77. doi: 10.15381/rivep.v24i1.1663

4. De Vivero L, Chavera A, Perales $R$, Fernández V. 2013. Frecuencia de neoplasias caninas en Lima: estudio retrospectivo en el periodo 1995-2006. Rev Inv Vet Perú 24: 182-188. doi: 10.15381/ rivep.v24i2.2487

5. Díaz M. 1990. Procesos neoplásicos en caninos: análisis estadístico periodo 19841989. Tesis de Médico Veterinario. Lima: Univ Nacional Mayor de San Marcos. $28 \mathrm{p}$.

6. Fajardo R, Alpízar A, Pérez L, Martínez J, Córdova E. 2013. Prevalence of tumors in dogs from the municipality of Toluca, México, from 2002 to 2008. Arch Med Vet 45: 305309. doi: 10.4067/S0301-732X2013000300011

7. Meuten D. 2002. Tumors of domestic animals. $4^{\mathrm{a}}$ ed. USA: Blackwell Publishing. $120 \mathrm{p}$.

8. Morris J. 2002. Oncología en pequeños animales. Buenos Aires, Argentina: Inter-Médica. $265 \mathrm{p}$.

9. Rodriguez J. 1978. Neoplasias en la especie canina. Estudio estadístico de 301 casos entre 1964-1977. Tesis de Médico Veterinario. Lima: Univ Nacional Mayor de San Marcos. 33 p.

10. San Martín MA. 2005. Neoplasias caninas: evaluación estadística periodo 1990-1994. Tesis de Médico Veterinario. Lima: Univ Nacional Mayor de San Marcos. 45 p.

11. Schmidt J, North S, Freeman K, Ramiro-Ibanez F. 2010. Canine paediatric oncology: retrospective assessment of 9522 tumours in dogs up to 12 months (1993-2008). Vet Comp Oncol 8: 283-292. doi: 10.1111/j.14765829.2010.00226.x 
12. Takano F. 1964. Estudio estadístico de 154 procesos neoplásicos en la especie canina. Tesis de Médico Veterinario. Lima: Univ Nacional Mayor de San Marcos. 51 p.
13. van den Hoven R. 2011. Air pollution and domestic animals. In: Air pollution new developments. Croatia: InTech. $p$ 179-202. 